\title{
Détermination de la structure tectonique de l'aquifère crayeux du littoral Nord Pas-de-Calais par prospection géophysique couplée à des observations par forage. Conséquence sur la répartition d'eau salée \\ Determination of tectonic structure in the chalk aquifer along the Nord Pas-de-Calais coast by geophysical prospecting coupled to observations by drilling. Consequences on the salted water distribution
}

\section{B. Louche et V. Hallet}

Volume 14, numéro 3, 2001

URI : https://id.erudit.org/iderudit/705420ar

DOI : https://doi.org/10.7202/705420ar

Aller au sommaire du numéro

Éditeur(s)

Université du Québec - INRS-Eau, Terre et Environnement (INRS-ETE)

ISSN

0992-7158 (imprimé)

1718-8598 (numérique)

Découvrir la revue

Citer cet article

Louche, B. \& Hallet, V. (2001). Détermination de la structure tectonique de l'aquifère crayeux du littoral Nord Pas-de-Calais par prospection géophysique couplée à des observations par forage. Conséquence sur la répartition d'eau salée. Revue des sciences de l'eau / Journal of Water Science, 14(3), 265-280. https://doi.org/10.7202/705420ar

\section{Résumé de l'article}

Sur le littoral du Nord - Pas-de-Calais (France), la Craie d'âge Crétacé supérieur constitue l'aquifère le plus exploité régionalement pour l'alimentation en eau tant potable qu'industrielle. Sur la frange la plus littorale, l'aquifère crayeux, semi-captif est affecté par de nombreuses failles. Dans certains secteurs, la profondeur de la craie et la localisation des failles, restent aléatoires suite au manque de forages et d'affleurements. Afin de palier à ces lacunes, trois méthodes géophysiques (sondages électriques et sismiques, profilage électromagnétique) ont été appliquées.

La synthèse des données obtenues a permis de démontrer que la craie est découpée en une série de compartiments par des accidents tectoniques présentant un affaissement vers le Sud et vers l'Ouest. L'interprétation des données géophysiques a également permis d'obtenir des informations sur la répartition de l'interface eau douce - eau salée au sein de l'aquifère crayeux.

Dans la région, le concept classique de biseau salé ne permet pas d'expliquer l'irrégularité spatiale des intrusions. L'étude semble démontrer que la répartition et l'extension des intrusions d'eau salée peuvent être corrélées avec la localisation des accidents tectoniques, les intrusions les plus éloignées du rivage se faisant au droit des zones faillées.

Ceci amène à proposer une nouvelle approche concernant l'extension des intrusions d'eau salée : elles seraient directement tributaires de la géométrie et des caractéristiques hydrodynamiques de l'aquifère crayeux, ces dernières étant fortement influencées par la présence des failles. 


\title{
Détermination de la structure tectonique de l'aquifère crayeux du littoral Nord-Pas-de-Calais par prospection géophysique couplée à des observations par forage. Conséquence sur la répartition d'eau salée
}

\section{Determination of tectonic structure in the chalk aquifer along the Nord-Pas-de-Calais coast by geophysical prospecting coupled to observations by drilling. Consequences on the salted water distribution}

\section{B. LOUCHE ${ }^{1}$, V. HALLET ${ }^{2}$}

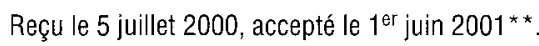

\section{SUMMARY}

\begin{abstract}
Along the North-Pas-de-Calais coast between a point south of Boutonnière du Boulonnais and the Authie River, Cretaceous rocks are limited to the east by a paleocliff that overlooks the Quaternary coastal and dune deposits. The chalk aquifer is connected to the Quaternary aquifers and further west to the sea water. Close to the seashore the top of the chalk plateau falls rapidly from $20 \mathrm{~m}$ above sea level to $20 \mathrm{~m}$ below sea level, causing the aquifer to grade from being unconfined in the east to confined in the west. This chalky aquifer is cut by several faults. A structural survey (LOUCHE et al., 1997) based on borehole data was carried out to evaluate whether the rate and direction of groundwater flow are controlled by the different faults. Two major fault groups striking $\mathrm{N} \mathrm{BO}^{\circ}$ and $\mathrm{N}^{\circ} 10^{\circ}$ have been revealed by the structural map, with a third minor group $\left(\mathrm{N} 160-170^{\circ}\right)$ to the north of the Canche. The two major groups, created during tectonic activity from the Palaeozoic to the present, are composed respectively of structures with general faulting directions of $\mathrm{N} 100-110^{\circ}$ and $\mathrm{N} 020-040^{\circ}$, previously described by COLBEAUX $e t$ al. (1993). Chalk depth and fault location are unknown in certain areas due to the lack of bore holes and outcrops. With the aim of overcoming these deficiencies, three traditional geophysical techniques (electrical sounding, seismic logging and electromagnetic profiling) were carried out between the Canche and Authie rivers in the coastal area, west of the chalky cliffs.
\end{abstract}

The interpretation of the geophysical results allows us to confirm the presence of previously located faults and identify new ones in the area where the chalk is overlain by Quaternary cover. The chalky aquifer is hence divided

1. LAMH, Université d'Artois, Faculté Jean Perrin, SP 18,62307 Lens cedex, France.

2. Département de géologie, Facultés universitaires Notre-Dame de la Paix, 61 rue de Bruxelles, 5000 Namur, Belgique.

* Correspondance. E-mail : barbara.louche@univ-artois.fr

* * Les commentaires seront reçus jusqu'au 14 août 2002. 
by the faults into a series of compartments that are inclined to the south or to the west. In addition, anomalies have been recorded in the fresh water - salt water interface on the Picardy continental shelf (MERCIER and BACROT, 1990). These anomalous zones are aligned along the northern extension of the sub N-S faults proposed by MENNESSIER and BEUN (1980), located a few kilometres to the south. Based on indirect evidence, these latter authors interpreted these faults as being responsible for considerable displacement of a major part of the Quaternary deposits. Given the lack of direct observations on salt-water distribution, geophysical results, and more specifically the electrical soundings, are required to provide supplementary information in order to better understand this phenomenon. Furthermore, the study of the salt water location, using electrical soundings, points out the juxtaposition of deep saline intrusions, demonstrating that the concept of a homogeneous wedge-shaped body parallel to the seashore can no longer be used to describe this chalky aquifer.

In the Bas-Champs area, electromagnetic profiling and three electrical soundings provided additional information on the relation between tectonic events and saline water intrusion (figure 3 ). The electromagnetic profiling shows the presence of two faults (figure 6). The vertical faults have an apparent low electrical resistivity $(2.5 \Omega . \mathrm{m})$ indicating the presence of brackish or salt water. Electrical sounding carried out to the south of the electromagnetic profile (SE9) shows a decrease in the electrical resistivity from $12.5 \mathrm{~m}$ depth, also confirming the presence of salt water. The results of an electrical sounding located to the north of the electromagnetic profile (SE10) show that the ground is saturated with fresh water up to at least $27.2 \mathrm{~m}$ (resistivity of $90 \Omega . \mathrm{m})$. At $1 \mathrm{~km}$ distance, there is a juxtaposition of three sectors separated by faults filled up with salt water. However, an electrical sounding (SE2, figure 2) located near the sea showed that the ground is saturated with fresh water up to at least $52.7 \mathrm{~m}$ deep.

Using geological, hydrogeological and geophysical data correlations, between structural context and saltwater intrusion could be established and a new hypothesis for saltwater intrusion for this study area could be proposed. Intrusion is directly dependent on the geometry and the hydrodynamic characteristics of the chalky aquifer, as reflected by its heterogeneous nature.

Salt water seems to follow preferential intrusion directions corresponding to faults rather than the classic model of a salt wedge. These results suggest the hypothesis that the seawater intrusion distribution relates to the tectonic configuration of the aquifer. The chalky series is cut into hydrogeological compartments individualised by tectonic accidents, which are preferential axes for sea intrusion. The presence of a saltwater front located far from the coast, as indicated by electrical soundings, could be explained by the geological history of the maritime plain, where the Pleistocene and Holocene seas covered the maritime plain up to the fossil cliff (LOUCHE et al., 1998).

Key-words: chalk aquifer, unconfined aquifer, geophysical methods, tectonic structure, salt-water intrusion.

\section{RÉSUMÉ}

Sur le littoral du Nord-Pas-de-Calais (France), la craie d'âge Crétacé supérieur constitue l'aquifère le plus exploité régionalement pour l'alimentation en eau tant potable qu'industrielle. Sur la frange la plus littorale, l'aquifère crayeux, semi-captif est affecté par de nombreuses failles. Dans certains secteurs, la profondeur de la craie et la localisation des failles, restent aléatoires suite au manque de forages et d'affleurements. Afin de pallier ces lacunes, trois méthodes géophysiques (sondages électriques et sismiques, profilage électromagnétique) ont été appliquées. 
La synthèse des données obtenues a permis de démontrer que la craie est découpée en une série de compartiments par des accidents tectoniques présentant un affaissement vers le Sud et vers l'Ouest. L'interprétation des données géophysiques a également permis d'obtenir des informations sur la répartition de l'interface eau douce - eau salée au sein de l'aquifère crayeux.

Dans la région, le concept classique de biseau salé ne permet pas d'expliquer l'irrégularité spatiale des intrusions. L'étude semble démontrer que la répartition et l'extension des intrusions d'eau salée peuvent être corrélées avec la localisation des accidents tectoniques, les intrusions les plus éloignées du rivage se faisant au droit des zones faillées.

Ceci amène à proposer une nouvelle approche concernant l'extension des intrusions d'eau salée : elles seraient directement tributaires de la géométrie et des caractéristiques hydrodynamiques de l'aquifère crayeux, ces dernières étant fortement influencées par la présence des failles.

Mots clés : aquifère crayeux, nappe semi-captive, prospection géophysique, structure tectonique, intrusion d'eau salée.

\section{1 - INTRODUCTION}

Depuis une dizaine d'années la zone littorale de la région Nord-Pas-deCalais est soumise à un développement économique relativement important. La connaissance de la ressource en eau devient nécessaire autant pour l'exploitation que pour la préservation.

Depuis le Cénomanien (base du Crétacé supérieur), époque à partir de laquelle s'est déposé le faciès crayeux dans le nord de la France, une succession d'événements tectoniques a permis le dépôt et la répartition de différentes formations tertiaires puis quaternaires. Entre la Canche et l'Authie (figure 1), la craie affleure à l'Est des falaises mortes rectilignes, entaillées de nombreux vallons secs qui sont séparées du massif dunaire par une plaine littorale (zone des Bas-Champs). Au niveau de celle-ci, le Sénonien est dans la majeure partie du secteur directement au contact des dépôts quaternaires marins. D'un point de vue hydraulique, cette nappe est libre à l'Est de la falaise fossile et semi-captive à l'Ouest.

La craie est affectée par une série de discontinuités ayant un rôle important sur les écoulements au sein de l'aquifère. Une étude structurale, du sud de la Boutonnière du Boulonnais à l'Authie (LOUCHE et al., 1997) avait permis de définir la géométrie de l'aquifère crayeux. Néanmoins sur la frange littorale, la profondeur de la craie et la localisation de failles restent imprécises par suite du manque de forages et d'affleurements. Afin de préciser la localisation de certaines failles lorsque la craie est sous recouvrement quaternaire, une campagne géophysique (sismique réfraction, prospection électrique et traînés électromagnétiques) a été réalisée dans une zone située entre Merlimont et Berck (figure 1).

Parallèlement, étant en bordure littorale, il existe une interface eau douceeau salée, dont la configuration était jusqu'alors inconnue. Les méthodes géophysiques appliquées pour l'étude structurale (plus particulièrement la 


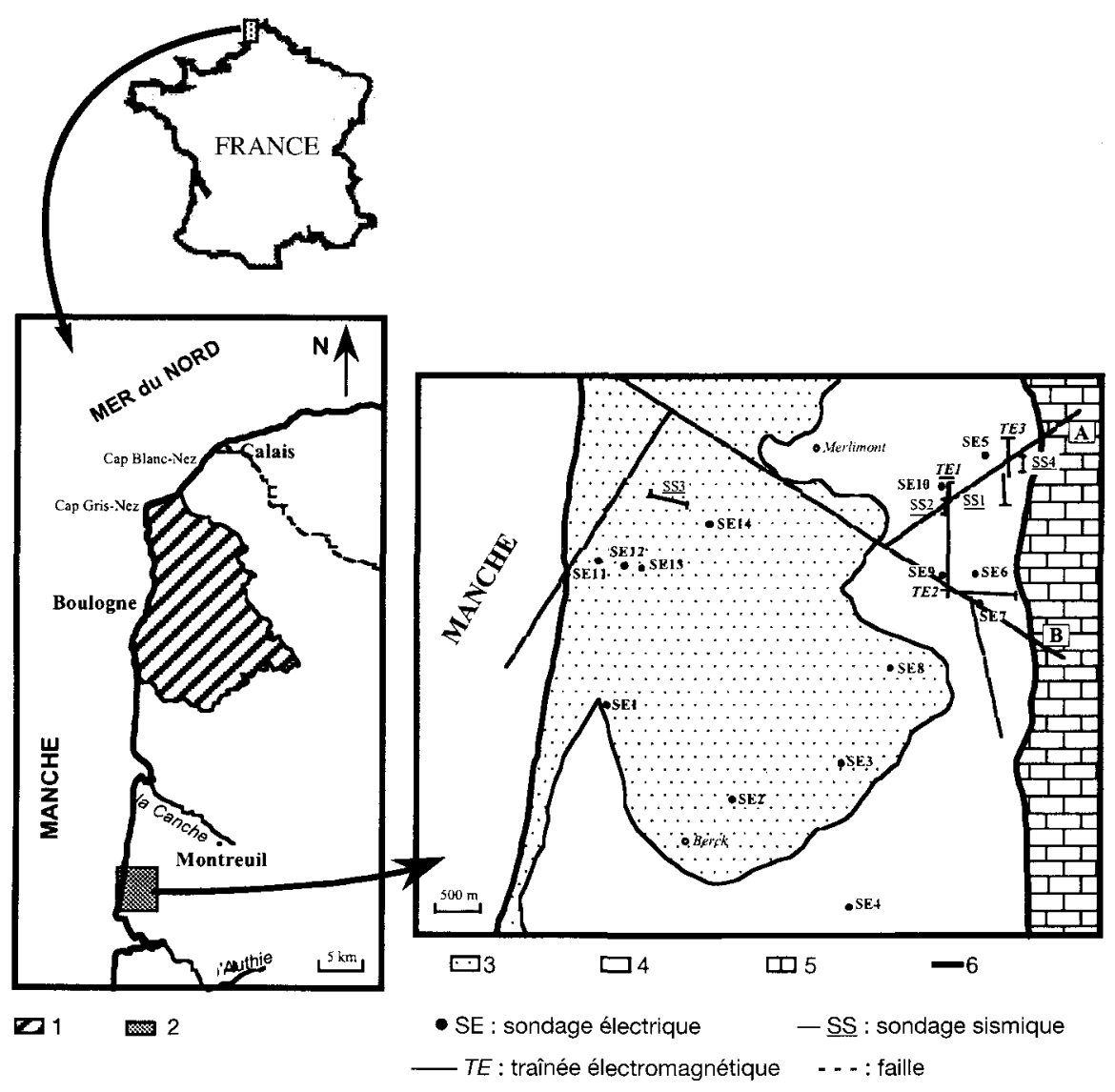

Figure 1 Localisation du secteur d'étude et détermination des essais géophysiques.

1 : boutonnière du Boulonnais $; 2,3:$ secteur d'étude ; $4:$ dunes littorales ; 5 : BasChamps ; 6 : plateau crayeux ; 7 : falaise fossile.

Location of the studied site and determination of geophysical tests.

1: boutonnière du Boulonnais; 2:studied site; 3: coastal dunes; 4: Bas-Champs; 5: chalky plateau; 6: paleocliff.

prospection électrique) ont permis d'obtenir des informations à ce sujet. Ces résultats ont été couplés avec les résultats d'analyses en chlorures réalisées dans les forages disponibles. Une confrontation des résultats de l'étude structurale et de la localisation de l'interface eau douce-eau salée a permis de proposer un nouveau concept concernant les écoulements au sein de l'aquifère crayeux. 


\section{2 - STRUCTURE DE LA CRAIE}

\subsection{Données de forages}

COLBEAUX et al. (1993) ont démontré que dans le Boulonnais, les structures présentes sont liées à une activité tectonique du Paléozoïque à nos jours, dont les directions principales de fracturation sont $\mathrm{N} 100-110^{\circ}$ et $\mathrm{NO20}-040^{\circ}$, et les directions secondaires N060-070 et $\mathrm{N} 120-130^{\circ}$.

Dans tout le secteur d'étude, le plateau crayeux s'arrête subitement contre les dépôts quaternaires le long d'une ligne brisée communément appelée "falaise morte ". Pour les auteurs ayant travaillé dans ce secteur (DE LAMMERVILLE, 1969 ; GUÉRIN, 1966), la plate-forme sénonienne crayeuse présente une surface irrégulière, modelée par l'érosion subaérienne pendant la période d'émersion qui a précédé le dépôt du Quaternaire. De plus, la cote du toit de la craie sous le Quaternaire, obtenue grâce aux forages, présente des variations verticales rapides (avec un gradient de $10 \%$ ) en allant vers l'Ouest.

Une carte structurale réalisée grâce aux données de sondages disponibles au BRGM (LOUCHE et al., 1997) a montré que, dans l'ensemble du secteur, les deux familles d'accidents décrits généralement dans les séries crétacées du

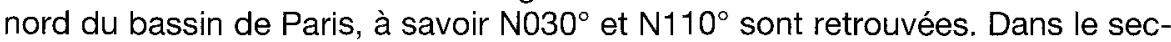
teur situé entre la Canche et l'Authie, une famille supplémentaire N160-170 a été mise en évidence. Ces accidents étant de plus faible extension, on peut expliquer leur absence ou leur non détection dans le reste du secteur d'étude par un nombre trop faible de forages.

Ces différents accidents séparent le secteur en plusieurs compartiments avec un décalage géologique très important dans la partie méridionale entre la partie au nord de la Canche, qui est surélevée, et la partie sud.

Ces différents compartiments sont découpés en compartiments plus petits avec un affaissement vers l'Ouest. L'ensemble du secteur d'étude est donc une structure en horst et graben.

\subsection{Résultats géophysiques}

Afin de confirmer le tracé des failles dans la zone des Bas-Champs, trois méthodes géophysiques ont été employées en plaine maritime en 1996, entre Merlimont et Berck. Elles consistent en :

- des sondages électriques pour déterminer la nature et l'épaisseur des dépôts tertiaires et quaternaires (résistivimètre ABEM SAS 300) avec le dispositif Schlumberger. Les valeurs de résistivités obtenues permettent de différencier aisément les formations tertiaires et quaternaires des formations crayeuses, ainsi que de détecter la présence ou non d'eau salée;

- des traînés électromagnétiques pour localiser les failles (zones de plus faible résistivités électriques), le matériel utilisé est un Geonics EM34 avec une distance entre les boucles de $40 \mathrm{~m}$;

- des sondages de sismique réfraction afin de localiser et de déterminer le pendage éventuel du toit de la craie, le contraste de vitesses sismiques entre les terrains tertiaires et quaternaires (140 à $1300 \mathrm{~m} / \mathrm{s})$ et les craies ( $2000 \mathrm{~m} / \mathrm{s}$ ) étant bien marqué. Ceci a été réalisé grâce à un équipement Geometrics ES 1225. 

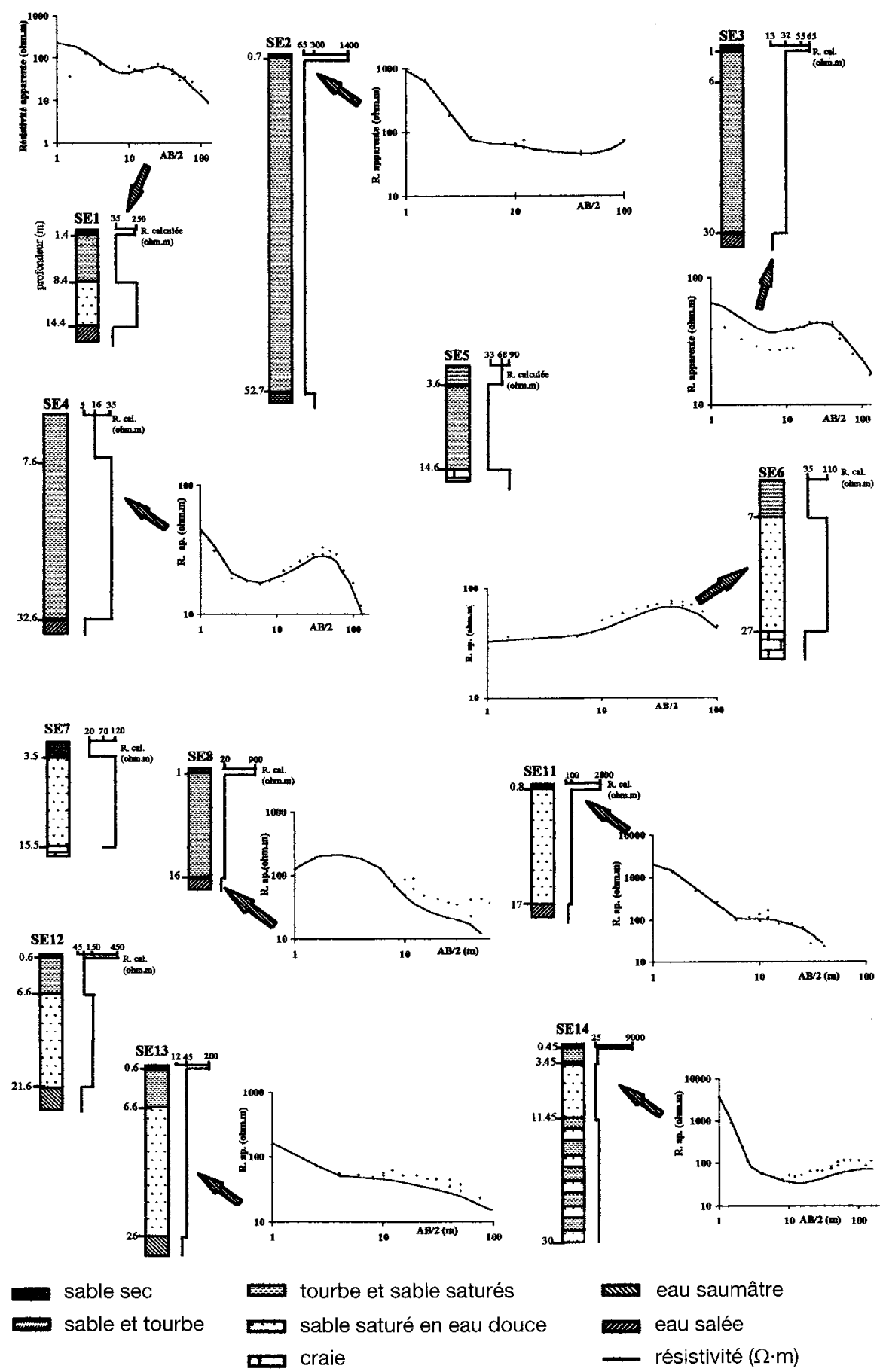

Figure 2 Interprétation géologique des sondages électriques.

Geological interpretation inferred from electrical soundings. 
Le choix de ces différentes méthodes a été effectué en fonction de leurs caractéristiques. En effet, la vitesse sismique obtenue par la sismique réfraction permet d'obtenir une coupe lithologique du milieu ainsi que des informations tectoniques. La prospection électrique est, quant à elle, une méthode simple et peu coûteuse, qui permet d'obtenir grâce au calcul des résistivités apparentes un log stratigraphique et l'interface eau douce-eau salée. Le trainé électromagnétique est une méthode très rapide pour déceler la localisation d'anomalies (failles). Cette méthode est donc utilisée pour localiser la zone où sera réalisée la sismique réfraction.

La localisation de ces divers profils et sondages est notée sur la figure 1. L'ensemble des points de mesure étant situés à une altitude de l'ordre de $5 \mathrm{~m}$ NGF, les résultats sont donnés en profondeur.

\subsubsection{Prospection électrique}

Entre Merlimont et Berck, cinq sondages ont fourni des renseignements sur la localisation du toit de la craie (figure 2). Afin d'obtenir une corrélation résistivité-géologie, le sondage SE5 a été effectué à proximité d'un forage pour lequel la coupe géologique était disponible. Au niveau du sondage SE2, la craie est détectée vers $50 \mathrm{~m}$ de profondeur, à $15 \mathrm{~m}$ au droit des sondages SE5 et $\mathrm{SE} 7$, à $27 \mathrm{~m}$ pour le sondage SE6 et à $7,2 \mathrm{~m}$ pour le SE10. Ces différentes données montrent une très grande variabilité de la cote du toit de la craie sur un secteur relativement restreint. Pour les autres sondages, le toit de la craie n'a pu être localisé du fait de la présence d'eau salée.

\subsubsection{Prospection électromagnétique}

Trois traînés électromagnétiques, effectués dans les Bas-Champs, ont pour objet de confirmer l'existence de deux axes de faille ( $A$ et $B$ ), préalablement localisés à partir de forages (figure 1).

Le traîné électromagnétique TE1 (figure 3) montre l'existence de trois zones de résistivités apparentes distinctes. L'augmentation de résistivité apparente, observée du Sud vers le Nord, pourrait être liée à une remontée du substratum crayeux (la craie étant plus résistive que les silts et la tourbe sus-jacents). Ces trois compartiments sont probablement séparées par des failles au droit desquelles de très faibles résistivités apparentes sont observées.

Afin de confirmer la localisation d'autres failles préalablement définies grâce aux données de forages, les traînés électromagnétiques TE2 et TE3 ont été réalisés.

Le profil TE2 (figure 4) permet la détermination de quatre zones distinctes, avec d'Ouest en Est :

- zone 1 : elle est caractérisée par des terrains de faible résistivité apparente $(<40 \Omega \cdot \mathrm{m})$ signalant la présence d'eau saumâtre, mise en évidence par le sondage électrique SE9 ;

- zone 2 : dans laquelle les valeurs de résistivité apparente augmentent régulièrement vers l'Est ;

- zone 3 : où les terrains superficiels sont de plus forte résistivité apparente du fait de la présence d'une couche épaisse de $12 \mathrm{~m}$, ayant une résistivité apparente de $120 \Omega \cdot m$ (SE6 et SE7) et pouvant être dus à la présence de 

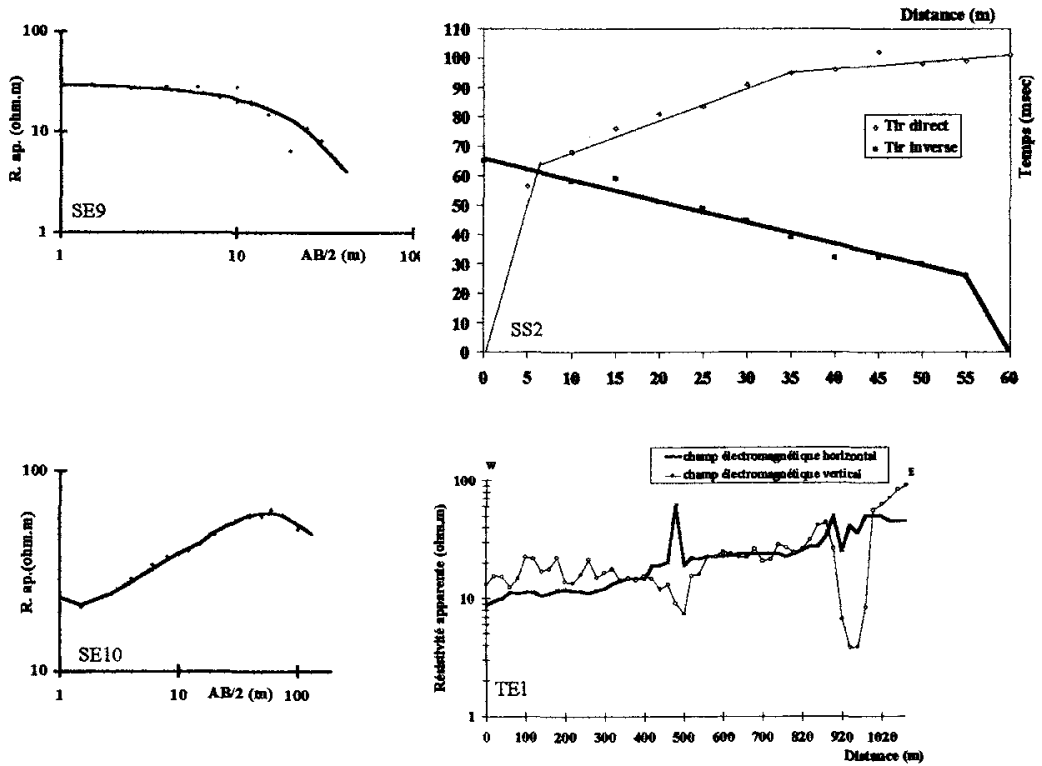

S

$\mathrm{N}$
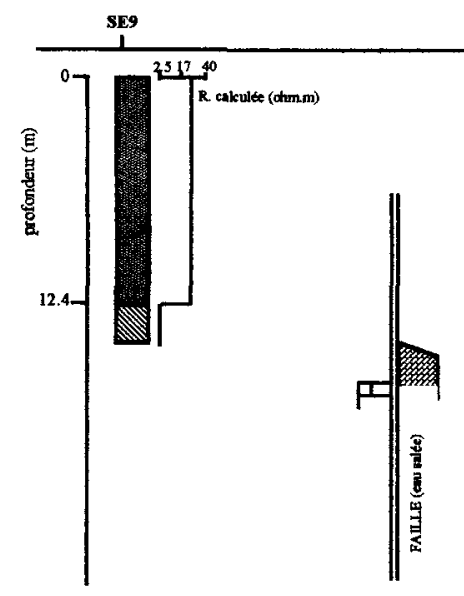

TE1 $40 \exists$ R calcille (otom m)

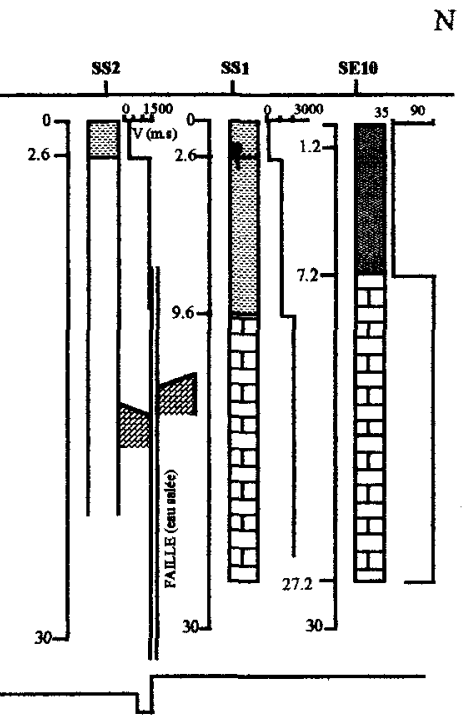

$$
\begin{aligned}
& \text { tourbe } \\
& \text { argile, tourbe et sable } \\
& \text { craie } \\
& \text { eau salée ou saumâtre }
\end{aligned}
$$

$$
\text { SE : sondage électrique }
$$$$
\text { SS : sondage sismique }
$$$$
\text { TE : trainé électromagnétique }
$$

$\longrightarrow 100 \mathrm{~m}$

Figure 3 Essais géophysiques et leur traduction géologique le long du traîné électromagnétique TE1.

Geophysical tests and their geological interpretation along electromagnetic profile TE1. 


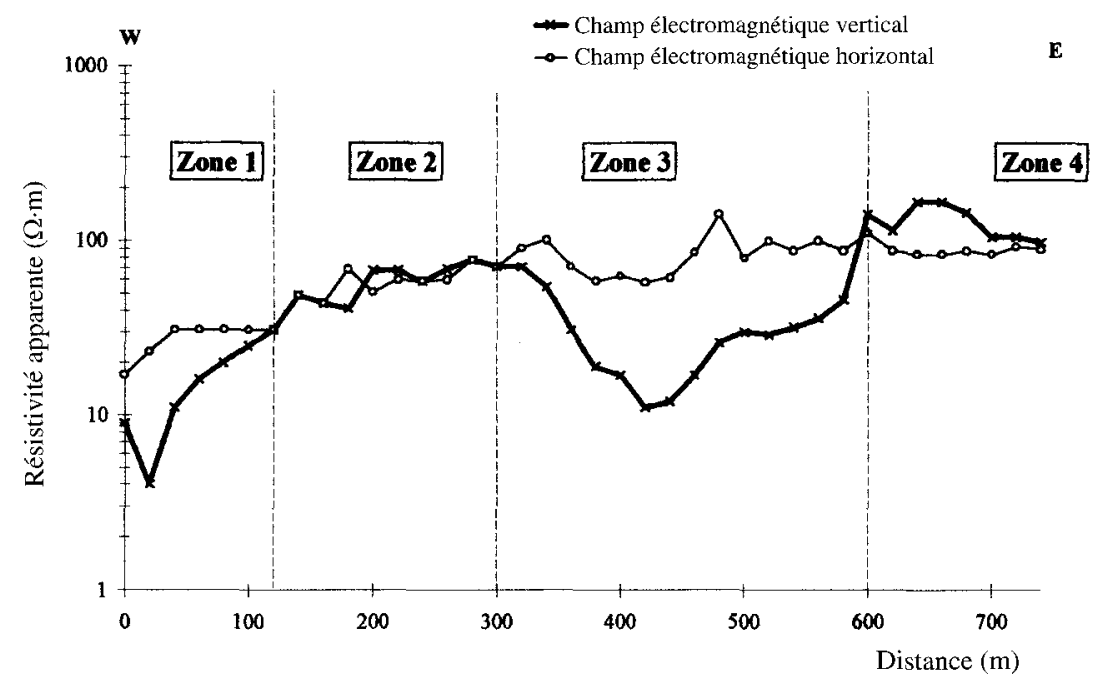

Figure 4 Profil électromagnétique (TE2) Ouest-Est.

Electromagnetic profile (TE2) West-East.

terrains pléistocènes caractérisés par des galets de silex englobés dans une matrice sableuse à crayeuse ;

- zone 4 : de très forte résistivité apparente ; les formations pléistocènes disparaissent et la craie subaffleure.

Ce profil électromagnétique montre que les limites entre ces quatre zones sont très nettes, indiquant des changements importants du contexte géologique pouvant être liés à la présence de failles.

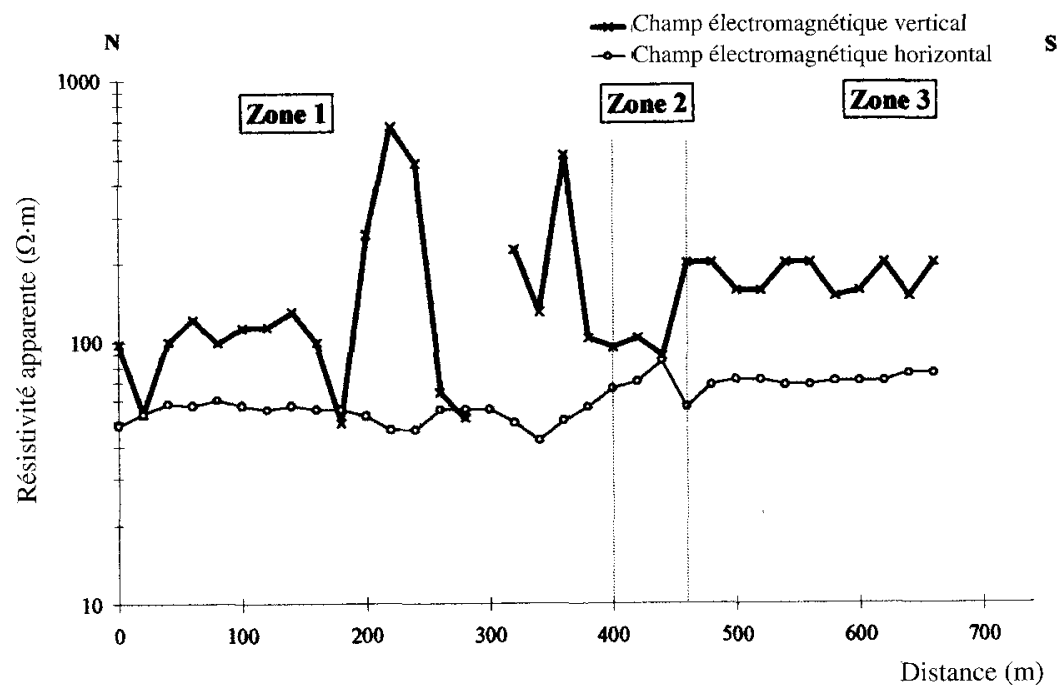

Figure 5 Profil électromagnétique (TE3) Nord-Sud.

Electromagnetic profile (TE3) North-South. 
Enfin pour le traîné TE3 (figure 5), les résultats obtenus pour le champ électromagnétique vertical mettent en évidence deux fortes anomalies. La différence de valeur entre l'EM34 en mode vertical et horizontal provient d'une différence de profondeur d'investigation (MC. NEILL, 1980). On peut remarquer la discontinuité du profil TE3 à $300 \mathrm{~m}$. Cette interruption est probablement liée à des valeurs très basses de conductivités qui rendent les mesures instables.

\subsubsection{Prospection sismique}

Quatre sondages de sismique réfraction ont été réalisés : trois dans les Bas-Champs et un au sein du massif dunaire. Les vitesses sismiques déterminées pour les différents matériaux sont les suivantes:

- tourbe sèche : $90-150 \mathrm{~m} . \mathrm{s}^{-1}$;

- tourbe saturée : $650-900 \mathrm{~m} . \mathrm{s}^{-1}$;

- sable sec : $700 \mathrm{~m} . \mathrm{s}^{-1}$;

- sable saturé : $1500-1600 \mathrm{~m} . \mathrm{s}^{-1}$;

- craie : 2 200-2 500 m.s ${ }^{-1}$.

Dans la plaine interdunaire, le sondage (SS3) a permis la détermination du toit de la craie à environ $35 \mathrm{~m}$ avec une inclinaison vers l'Ouest.

Dans les Bas-Champs (figure 3), le toit de la craie a été rencontré vers $9,6 \mathrm{~m}$ au droit du sondage SS1, plus profondément $(15 \mathrm{~m})$ au sondage SS2 et au niveau du sondage SS4 (figure 1), la craie est subaffleurante (entre 2 et $5 \mathrm{~m}$ ).

De plus au niveau du sondage SS2 (figure 3), la différence entre les résultats obtenus avec les tirs directs et les tirs inverses pourrait être due à l'inclinaison du toit du substratum qui présenterait un pendage Nord. En revanche le pendage du toit de la craie serait vers le Sud dans la zone où a été effectué le sondage SS1.

\subsection{Synthèse des résultats géophysiques}

\subsubsection{Structures}

La synthèse des résultats obtenus par l'utilisation des trois techniques géophysiques le long du traîné électromagnétique TE1 (figure 3) a permis de confirmer la présence de trois parties distinctes séparées par deux anomalies de très faible résistivité correspondant à des failles. L'augmentation de la résistivité des terrains du Sud vers le Nord est la réponse d'une remontée du substratum crayeux. Cette hypothèse est tout à fait en accord avec la carte structurale. Le sondage sismique SS1, réalisé au Nord du profil, montre que le toit de la craie se trouve vers $9 \mathrm{~m}$. Plus au Sud (sondage sismique SS2), le toit de la craie se situerait vers $15 \mathrm{~m}$. Une comparaison de la localisation de ces deux accidents le long de ce premier traîné avec l'emplacement des anomalies déterminées par les traînés électromagnétiques TE2 et TE3, permet alors de réajuster la direction et le tracé des failles déduites de l'interprétation des données de forages (figure 6). En effet, compte tenu du nombre restreint de forages disponibles pour la réalisation de la carte structurale, il existait un certain nombre d'incertitudes en ce qui concerne le positionnement de ces failles. De manière globale, on retrouve les trois failles décrites dans ce secteur, la faille $n^{\circ} 1$ 
confirmée par la géophysique, la $n^{\circ} 2$ prolongée vers le Nord et la faille $n^{\circ} 3$ affectée par la $n^{\circ} 2$ et légèrement décalée vers le Sud. De plus, deux failles supplémentaires $\left(n^{\circ} 4\right.$ et 5 ) d'orientation $N 170^{\circ}$ ont dû être tracées à partir des données géophysiques obtenues dans cette zone et plus à l'Est.

À proximité de la côte, le toit de la craie a été mis en évidence à $37 \mathrm{~m}$ de profondeur, avec un regard vers l'Ouest, ce qui confirme bien la configuration de la craie décrite lors de l'interprétation des données de sondages, en blocs affaissés d'Est en Ouest.

À partir des résultats de forages et de la géophysique, il a pu être démontré que la série crayeuse est découpée en une série de compartiments par l'intermédiaire d'accidents tectoniques, avec un affaissement général vers l'Ouest. Le toit de la craie se situe vers une profondeur de $5 \mathrm{~m}$ dans les Bas-Champs et de $35 \mathrm{~m}$ sous la plaine interdunaire. Compte tenu de la répartition des résultats géophysiques, on ne peut préciser que localement le tracé de certaines failles.

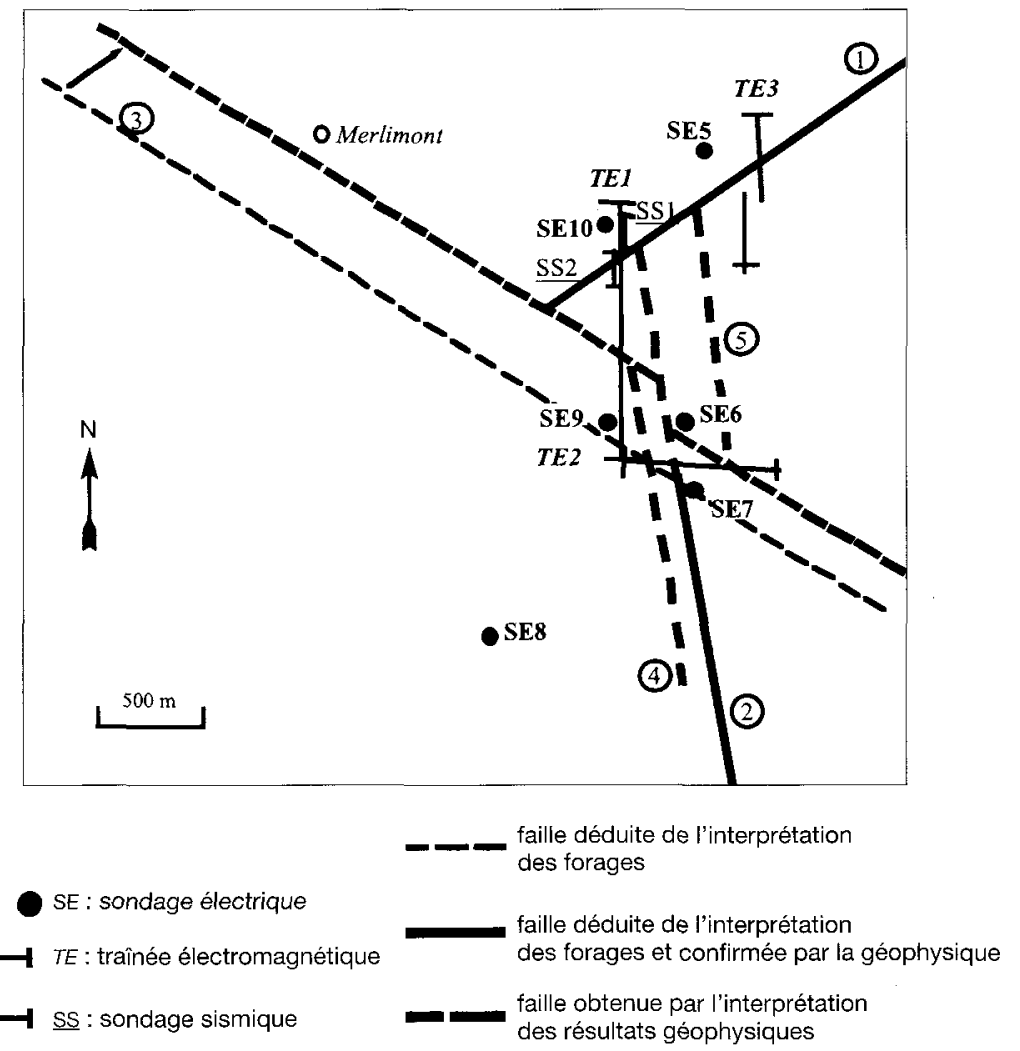

Figure 6 Localisation des failles dans la zone Nord-Est des Bas-Champs.

Fault location in the Bas-Champs North-East area. 


\subsubsection{Répartition de l'interface eau douce-eau salée}

Les sondages électriques et les profils électromagnétiques ont également apporté un complément d'information pour la compréhension de la répartition de la limite eau douce-eau salée.

Les sondages électriques réalisés dans le massif dunaire ont été orientés Nord-Sud (parallèlement à la côte). Ils avaient pour objet de localiser la limite entre les terrains saturés en eau douce et en eau salée, et d'en déterminer leur nature.

La synthèse des résultats obtenus grâce à la réalisation de sondages électriques permet de proposer une coupe d'orientation Est-Ouest (figure 7). Cette coupe montre que dans le secteur Ouest, la répartition des différents types d'eau (salée, saumâtre et douce) est en accord avec le concept de biseau salé, classiquement décrit en région littorale soit, de la côte vers l'intérieur des terres, une succession d'eaux salée, saumâtre et douce. En effet, les formations sont envahies d'eau salée au droit du sondage SE11, puis d'eau saumâtre au droit des sondages SE12 et SE13 alors que l'eau est douce jusqu'au moins $30 \mathrm{~m}$ de profondeur au niveau du SE14.

W

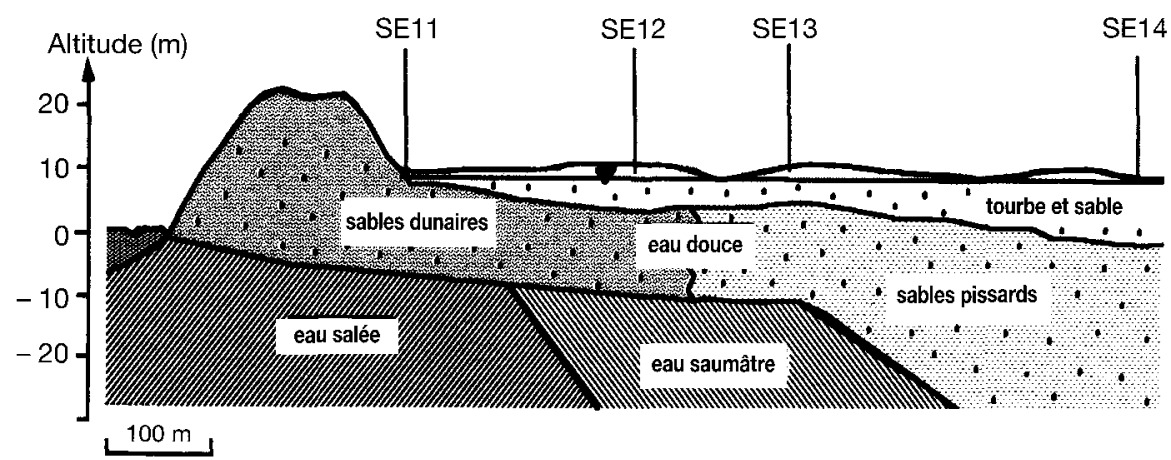

Figure 7 Coupe dans la réserve biologique de Merlimont mettant en évidence la disposition des différentes masses d'eaux : douce, saumâtre et salée.

Cross-section of the Merlimont biologic reserve providing a better explanation for the pattern of water distribution: fresh, brackish and salty.

À l'échelle régionale, la configuration classique du biseau salé n'est pas systématiquement observée. Les différents sondages électriques (figures 2 et 3) ont mis en évidence la présence d'eau salée ou saumâtre aux profondeurs suivantes:

- SE1, l'interface eau douce-eau salée a été atteinte à $14 \mathrm{~m}$ de profondeur ;

- SE2, la présence d'eau douce a été observée jusqu'à $53 \mathrm{~m}$ de profondeur ;

- à l'Est, c'est-à-dire à l'intérieur des terres, l'eau saumâtre a été atteinte à $30 \mathrm{~m}$ au niveau du sondage SE3, à 12,4 pour le sondage SE9 et à $27 \mathrm{~m}$ au droit du sondage SE6 ; 
- compte tenu des valeurs de résistivités au droit des sondages SE8 et SE4, l'eau est salée à respectivement 16 et $32 \mathrm{~m}$.

Ces constatations peuvent être confortées par l'analyse des concentrations en chlorures dans les forages disponibles dans cette zone (figure 8). Celles-ci varient sur l'ensemble des points de prélèvement de $35 \mathrm{mg} / \mathrm{l}$ (sur le coteau crayeux ainsi qu'à la limite de celui-ci avec la plaine picarde) à $760 \mathrm{mg} / \mathrm{l}$. II faut noter la très faible concentration en chlorure $(35 \mathrm{mg} / \mathrm{l})$ pour le point situé le plus à l'Ouest, ce qui est tout à fait en accord avec les données issues de la géophysique (sondage électrique SE14). En revanche, quatre forages implantés à l'intérieur des terres sont salés, ce qui confirme les résultats des différents sondages électriques réalisés dans ce secteur.

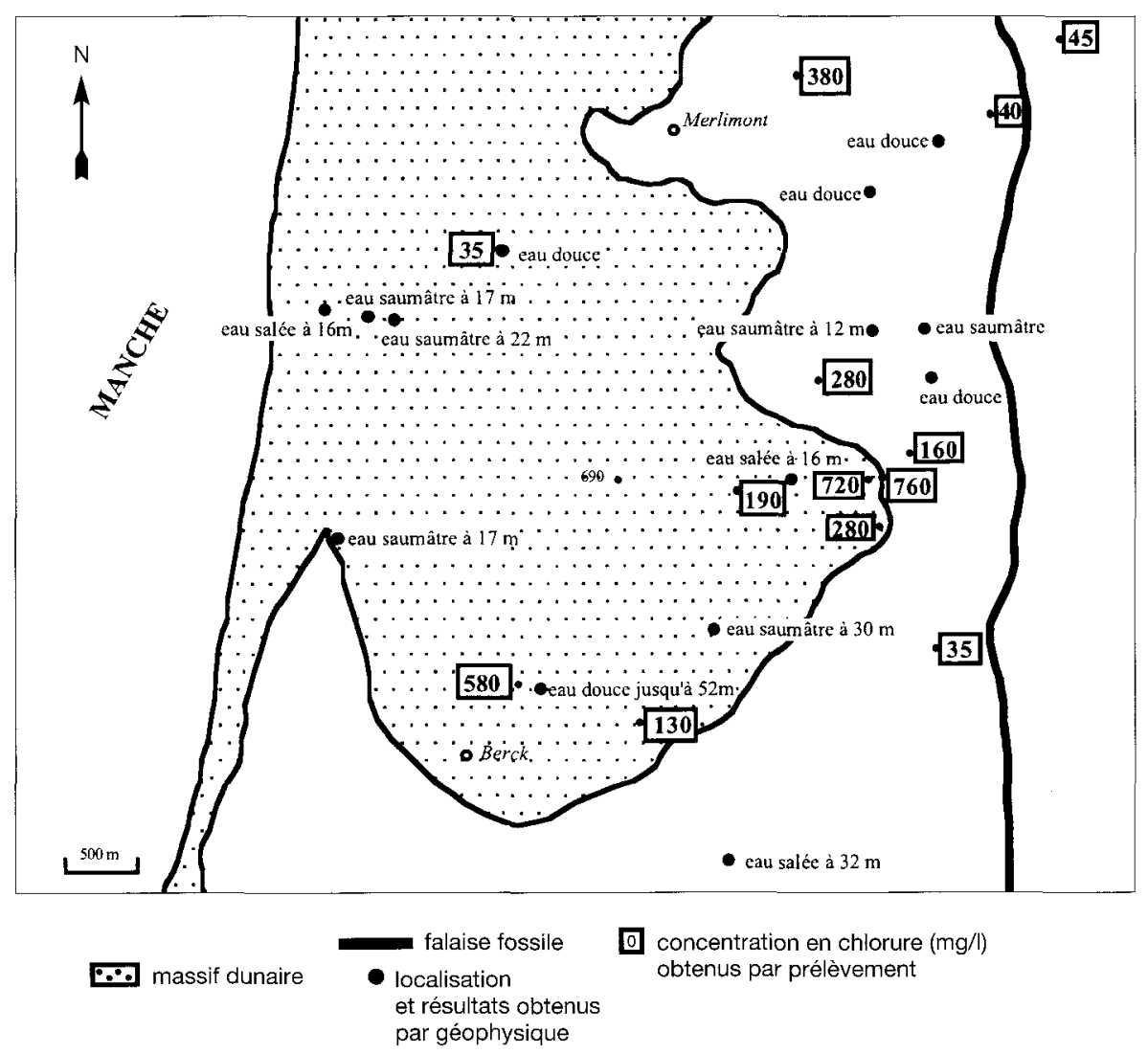

Figure 8 Répartition des différents types d'eau (douce-saumâtre-salée) entre Merlimont et Berck.

Distribution of groundwater (fresh, brackish, salty) between Merlimont and Berck.

L'étude géophysique démontre bien l'hétérogénéité spatiale de la répartition de l'eau salée à saumâtre dans cette zone littorale. À proximité de la côte, le sondage SE14 est dépourvu d'eau saumâtre jusqu'au moins $30 \mathrm{~m}$ de profondeur alors que dans les Bas-Champs l'eau saumâtre a été détectée à 12,5 m 
de profondeur au sondage SE9. De plus, le sondage électrique SE2 révèle l'absence d'eau saumâtre ou salée jusqu'à $53 \mathrm{~m}$ de profondeur, alors que les zones adjacentes sont saturées d'eau saumâtre voire salée.

Au vu de ces informations, le concept de biseau salé ne peut, à lui seul, expliquer cette répartition.

\subsection{Comparaison entre la localisation des intrusions et la structure de l'aquifère}

Les résultats des études hydrogéologiques et géophysiques réalisées dans la région de Merlimont, permettent de mettre en évidence la complexité de la répartition de l'interface eau douce-eau salée. La répartition en dent de scie, de l'eau salée, douce et saumâtre, indépendamment de la profondeur des forages est en désaccord avec le concept du biseau salé. Des hétérogénéités de l'interface eau douce-eau salée ont d'ailleurs été démontrées (MERCIER et BACROT, 1990) dans la plaine maritime picarde. Celles-ci s'alignent dans le prolongement septentrional de failles subméridiennes, proposées, quelques kilomètres plus au sud, par MENNESSIER et BEUN (1980). Ces failles décaleraient sensiblement une grande partie du Quaternaire. Ceci suppose une intrusion d'eau marine à la faveur de la fracturation. Cette hypothèse d'intrusion marine par l'intermédiaire de failles permettrait d'expliquer la présence d'eau salée à une distance relativement éloignée par rapport à la mer et la présence d'eau douce dans des forages proches de la mer mais situés hors des zones faillées.

Dans les Bas-Champs, les données géophysiques, confirmées par les logs de forage permettent de proposer un modèle où la zone d'étude est séparée en sept compartiments par six failles (figure 9). La série crayeuse est donc découpée en une série de compartiments, par l'intermédiaire d'accidents tectoniques, avec un affaissement vers l'Ouest, comme le confirme le sondage SS3 où le toit de la craie a été détecté vers $35 \mathrm{~m}$.

L'étude de la répartition de l'interface eau douce-eau salée au sein de l'aquifère crayeux semi-captif a permis de mettre en évidence la complexité du système et l'influence des failles sur les intrusions salées. Perpendiculairement aux accidents d'orientation $\mathrm{N} 40^{\circ}$ et $\mathrm{N} 120^{\circ}$, le traîné électromagnétique TE1 (figure 3), présenté précédemment a démontré la juxtaposition de trois compartiments séparés par des failles saturées d'eau salée avec la présence d'eau saumâtre à faible profondeur dans le compartiment Sud. Les intrusions sont conditionnées d'une part par la structure et la géométrie de la craie et d'autre part par le degré de fracturation de chaque compartiment hydrogéologique et donc par la perméabilité du milieu.

En effet, une comparaison entre la répartition des perméabilités obtenues par modélisation de l'aquifère crayeux et la carte structurale a conduit à ne plus considérer l'aquifère comme un système unique, mais comme un pavage de compartiments séparés par des grands accidents tectoniques d'échelle plurikilométrique, compartiments eux même subdivisés en sous-compartiments au sein desquels la perméabilité est relativement homogène (LOUCHE et al.,1997). Les résultats obtenus dans l'étude de la répartition de l'eau salée dans ce secteur permet alors de confirmer ceux obtenus par la modélisation. Néanmoins, l'échelle d'étude dans la modélisation étant différente de celle de l'étude de la répartition de l'interface eau douce-eau salée, il n'est pas possible 


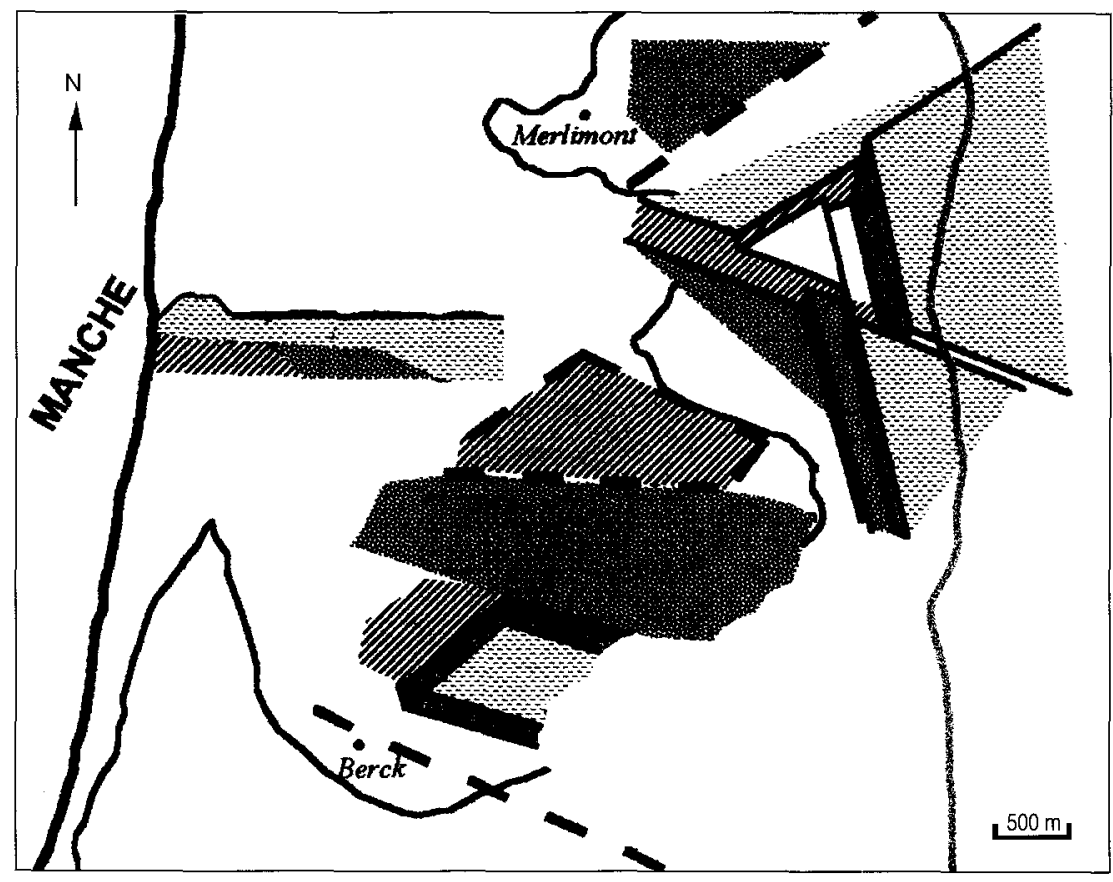

eau douce

Figure 9 Relation entre géométrie de l'aquifère crayeux et la localisation des eaux saumâtres et salées.

Relation between the geometry of the chalk aquifer and the distribution of brackish and salt water.

de mettre en évidence la relation entre la perméabilité de l'aquifère et le type d'eau rencontré.

\section{3 - CONCLUSION}

L'utilisation conjointe des méthodes d'investigation hydrogéologique et géophysique, a permis de préciser non seulement la géométrie des formations crayeuses de la région de Merlimont (Nord de la France) et les structures les affectant, mais également l'extension des intrusions d'eau salée. Dans cette région, l'aquifère crayeux est subdivisé en une série de blocs à degré de fissuration variable séparés par des accidents tectoniques. La localisation de points de contact eau douce-eau salée, a mis en évidence la juxtaposition de zones d'intrusion d'eau salée plus ou moins pénétrantes démontrant que le concept classique du biseau d'eau salée n'est pas applicable dans la région. 
La corrélation entre l'extension des intrusions et la géométrie de l'aquifère crayeux, permet de mieux comprendre la localisation de l'eau salée au sein de ce dernier : la répartition de l'eau salée est tributaire de la géométrie de l'aquifère. Ce dernier est découpé en une suite de compartiments ou blocs hydrogéologiques individualisés par des accidents tectoniques qui constituent des axes préférentiels d'intrusion ou de stockage d'eau marine. Les blocs présentent des degrés de fissuration variables et sont plus ou moins aquifères selon le développement de la fissuration. La localisation des intrusions d'eau salée est directement influencée par la répartition des discontinuités structurales. En vue de préciser le cadre hydrogéologique de la région de Merlimont, il serait intéressant de réaliser une nouvelle campagne de sondages électriques selon un maillage systématique de $500 \times 500 \mathrm{~m}$.

En milieu littoral, une meilleure compréhension devrait permettre par la suite une gestion quantitative et qualitative des aquifères. Ceci permettrait de proposer une implantation judicieuse des sites de captage et la détermination des capacités de pompage afin de préserver la nappe de toute contamination ultérieure par l'eau salée.

\section{RÉFÉRENCES BIBLIOGRAPHIQUES}

COLBEAUX J.P., DUPUIS CH., ROBASZYNSKI F., AUFFRET J.P., HAESAERTS P., SOMMÉ J., 1980. Le détroit du Pasde-Calais: un élément dans la tectonique de blocs de l'Europe nord-occidentale. Bull. Inf. Géol. Bassin Paris, 17, 41-54.

COLBEAUX J.P., SOMMÉ J., 1981. Fracturation du substrat crayeux et géomorphologie dans le nord de la France. Exemple de la feuille de Desvres à 1/50 000. Bull. Ass. Fr. Quaternaire, 1, 33-39.

COLBEAUX J.P., AMEDRO F., BERGERAT F.,BRACQ P., CRAMPON N., DELAY F., DUPUIS $\mathrm{CH}$., LAMOUROUX $\mathrm{CH}$., ROBASZYNSKI F., SOMMÉ J., VANDYCKE S., VIDIER J.P., 1993. Un enregistreur des épisodes tectoniques dans le bassin de Paris : le Boulonnais. Bull. Soc. Géol. France, 164 (1), 93-102.

GUÉRIN B., 1966. Contribution à l'étude hydrogéologique du bassin de la Canche. Thèse $3^{e}$ cycle Univ. Paris IV, $181 \mathrm{p}$.

LAMMERVILLE (DE) J.M., 1969. Contribution à l'étude hydrogéologique de la plaine du Marquenterre. Thèse $3^{e}$ cycle Univ. Paris, $84 \mathrm{p}$.

LOUCHE B., DELAY F., CARLIER E., 1997. Comparaison des caractéristiques hydrodynamiques et structurales de l'aquifère crayeux du littoral Nord-Pasde-Calais. Soc. Géol. France, 168 (4), 451-461.

LOUCHE B., CRAMPON N., BRACQ P., 1998. Qualité et comportement de l'aquifère crayeux sur le littoral Nord-Pas-deCalais. CR Acad. Sci., 327, 463-470.

MC NEILL J.,1980. Electromagnetic terrain conductivity measurement at low induction number, Tech. Note TN-6. Geonics limited.

MENNESSIER G., BEUN N., 1980. Géologie de la Picardie. CRDP éd., Amiens, $119 \mathrm{p}$.

MERCIER E., BACROT S., 1990. Note préliminaire sur des anomalies de l'interface eau douce/eau salée dans les plaines maritimes flamande et picarde. Ann. Soc. Géol. Nord., 205-210. 